\title{
POESÍA A DOS BANDAS: Innovación Docente a Través de la Literatura y la Traducción
}

Bruno Echauri Galván

Universidad de Alcalá (bruno.echauri@uah.es)

\section{Silvia García Hernández}

Universidad de Alcalá (silvia.garciah@uah.es)

\begin{abstract}
This paper shows the development of a Teaching Innovation Project carried out with some students of two different degrees: Hispanic Studies and Modern Languages and Translation, at the University of Alcalá. Our main objective here is to show how the project was conducted and the results obtained. The idea for the project emerged from a key aspect: the literary vocation of some students in both degrees. With this in mind, the creation of a bilingual collection of poems was proposed. Firstly, students in Hispanic Studies would create some poems that could be translated, or rather "recreated", by the Translation students. The main objectives of this interdisciplinary project are to promote creativity and increase students' motivation, as well as to reinforce both the use of English as a foreign language and the practice of literary translation by using original texts created by other classmates. By doing this, group work was also encouraged. Connected to collaborative work, another innovative element included in this project is the use of ICT, since students have worked together not only in face-to-face meetings, but also by means of new technologies such as Blackboard Collaborate, among others, which have enabled them to keep contact, and share documents and opinions in real time.
\end{abstract}

Keywords: poetry, literary translation, innovation, new technologies, creativity, motivation, group work.

\footnotetext{
Resumen

El presente trabajo muestra el desarrollo de un proyecto de innovación docente llevado a cabo con alumnos de dos grados, Estudios Hispánicos y Lenguas Modernas y Traducción, en la Universidad de Alcalá. El objetivo de estas páginas es exponer el desarrollo y resultados de este proyecto que surge de la visión de un aspecto fundamental: la vocación literaria de algunos alumnos de ambos grados. Partiendo de esta base, se propuso la realización de un poemario bilingüe en el que los alumnos del grado en Estudios Hispánicos crearan poemas que pudieran ser traducidos o, más bien, "re-creados", por parte de los alumnos traductores. Los objetivos principales de este proyecto interdisciplinar son los de fomentar la creatividad y aumentar la motivación de los alumnos de ambos grados, así
} 
como reforzar el uso de la lengua extranjera y la práctica de la traducción literaria con textos originales creados por otros compañeros, fomentando asi el trabajo colaborativo. Además, otro de los puntos innovadores de este proyecto es que los alumnos pudieran trabajar juntos a través de las nuevas tecnologías, utilizando, entre otras, la herramienta Blackboard Collaborate, que les permitiera estar en contacto, compartir documentos y opiniones en tiempo real.

Palabras clave: poesía, traducción literaria, innovación, nuevas tecnologías, creatividad, motivación, trabajo en grupo.

\section{Introducción}

El texto que ocupa las siguientes páginas presenta un proyecto de innovación docente realizado en la Universidad de Alcalá durante el curso 2016/2017 por cuatro profesores (el doctor Bruno Echauri Galván, la doctora Silvia García Hernández, la doctora Maya García de Vinuesa y el doctor Jonathan Sell) y veinte alumnos de dos grados diferentes: el segundo curso de Estudios Hispánicos (EEHH en adelante) y el tercer y cuarto curso de Lenguas Modernas y Traducción (LLMMyT en adelante).

La idea para la realización de este proyecto de innovación docente surge de dos pilares fundamentales: el talento y vocación literaria de algunos alumnos del grado en Estudios Hispánicos y la posibilidad de que la calidad de sus escritos redundara en su beneficio y en el de otros ámbitos de la universidad. En este sentido, la asignatura Traducción Literaria, Edición y Corrección de Textos, impartida por la profesora García de Vinuesa, ofrece la plataforma perfecta para una actividad transversal con estos alumnos.

Una vez escogidos los dos grupos con los que se pretende trabajar, el proyecto se organiza alrededor de un propósito tangible e ilusionante para todos los participantes: la creación de un poemario bilingüe en el par de lenguas inglés-español. Pero detrás de este fin hay también otros objetivos que pasan a detallarse a continuación.

\section{Objetivos y bases teóricas}

Al margen del poemario bilingüe mencionado en el apartado anterior, el proyecto de innovación que se está describiendo busca conseguir otra serie de objetivos. El siguiente listado los presenta en detalle, añadiendo justificaciones teóricas cuando se estima necesario:

\subsection{Objetivo 1}

Potenciar la creatividad de los alumnos: Subrayar la creatividad como factor a potenciar en el proceso de aprendizaje (también de una segunda lengua), como mecanismo de renovación y actualización de la enseñanza en todos los niveles y como motor de nuevas ideas e iniciativas es una reivindicación de muchos autores (De la Torre, 2009 o Gervilla

2017, Universitat Politècnica de València 
Castillo, 2003 entre otros). En esta línea, se busca canalizar el talento (literario en este caso) del alumnado de modo que no sea únicamente una actividad periférica de tiempo libre y pueda repercutir en su carrera universitaria.

En línea con lo anterior, uno de los principales objetivos que ayudaron a cohesionar al grupo de profesores participantes es el intento de que la creatividad vaya ganando espacio dentro del currículo de los grados de letras. Y no se trata únicamente de amenizar el proceso de aprendizaje: también se busca explotar algunas capacidades del alumno que no se ven potenciadas tradicionalmente, que los alumnos desarrollen una mente y una capacidad creativa determinada y que aquellos que ya las tienen, las perfeccionen al mismo tiempo que esto tiene una recompensa en sus calificaciones finales. Por ello, el trabajo de los traductores en este proyecto fue tomado en consideración en su nota final ${ }^{1}$.

\subsection{Objetivo 2}

Estimular la motivación del alumnado a través de la innovación: Autores como ChaínNavarro, Martínez-Solís y Sánchez-Baena (2008) constatan que la desmotivación es uno de los males de la educación superior en nuestro país, y es necesario hacerle frente. En este sentido, los mismos autores señalan que la innovación docente puede ser uno de los mecanismos para paliar el desencanto que en ocasiones invade a parte de los alumnos e incluso a los propios docentes. Nuestro proyecto apuesta por crear una actividad diferente con aspectos novedosos e innovadores como los siguientes:

- La naturaleza puramente creativa de la tarea.

- El trabajo de traducción con textos puramente originales.

- La colaboración entre alumnos de distintos cursos y grados, potenciando así el trabajo en grupo interdisciplinar.

- El uso de una herramienta virtual (Blackboard Collaborate) no utilizada con anterioridad y que permite compatibilizar el trabajo telemático con la interacción directa por videoconferencia.

- La publicación en papel de los poemas y traducciones de los alumnos.

\subsection{Objetivo 3}

Refuerzo de la segunda lengua: Este objetivo se divide a su vez en dos vertientes dependiendo del grupo meta. En el caso de EEHH, se busca dar continuidad al contacto con el inglés y aumentar el interés de los alumnos por esta lengua. Hay que tener en cuenta que en el caso de EEHH, su contacto en la universidad con una L2 termina tras el primer curso. En nuestra opinión, este tipo de actividades puede ayudar a mantener o incluso incrementar

\footnotetext{
1 Esto no se pudo hacer con los alumnos de EEHH por no cursar ninguna de las asignaturas impartidas por los profesores involucrados. Por ello, queremos destacar especialmente su entrega completamente altruista en la elaboración de este proyecto.
} 
su deseo de no abandonar el estudio de un segundo idioma al trabajar en un ámbito que les motiva como es el literario.

En el caso de LLMMyT, el proyecto se orienta al perfeccionamiento del idioma y a un mejor manejo de la lengua inglesa $\mathrm{y}$, especialmente, del lenguaje literario. Este refuerzo de la segunda lengua es lo que explica el uso de la traducción inversa en el proyecto. Bien aplicada, la traducción inversa puede reportar numerosos beneficios. Entre ellos, se cuenta un incremento de la competencia cultural del traductor, una menor posibilidad de malinterpretar el texto original y un mayor cuidado en el empleo de la lengua meta (Posey, 2009). En esta misma línea, traducir a una segunda lengua implica normalmente una mayor afinidad con el autor original del texto, algo que muchos traductores consideran fundamental, especialmente en el ámbito de la poesía (Posey, 2009).

Por otro lado, pese a que muchas veces la traducción inversa ha sido denostada, etiquetando sus resultados (generalmente a priori) de falta de naturalidad, autores como McAlister (citado en Posey, 2009), defienden que es posible traducir un texto hacia una segunda lengua y hacerlo de forma clara y correcta. En este caso, consideramos que el resultado encaja perfectamente con las palabras de McAlister, ya que creemos que la calidad de las traducciones es realmente alta, más si tenemos en cuenta que los traductores se enfrentaban a su primer desafío de este tipo. Pero, en este sentido, preferimos dejar que cada uno extraiga sus propias conclusiones tras leer atentamente los ejemplos que se presentan unas páginas más adelante.

\subsection{Objetivo 4}

Mejora de la enseñanza de la traducción poética: Otro de los propósitos de este proyecto es mejorar la docencia en el ámbito de la traducción poética, en especial en lo que respecta a alumnos que se adentran por primera vez en este ámbito. En este sentido, se solicita a los traductores que elaboren un comentario en el que expliquen los principales rasgos del poema original y meta, así como los desafíos fundamentales a los que se han enfrentado durante el proceso traductológico. Un análisis de dichos comentarios y la recopilación de las dudas planteadas en los distintos talleres permiten establecer las dificultades más comunes entre los estudiantes. Dichos problemas abren un amplio campo de investigación y pueden servir como base para determinar pautas y prácticas docentes más adecuadas a las necesidades de aquellos alumnos que contactan por primera vez con la traducción literaria. Las pautas que se establezcan podrán utilizarse en cursos posteriores para mejorar el proceso de enseñanza y de aprendizaje en la sección de traducción poética de la asignatura de Traducción Literaria.

\subsection{Objetivo 5}

Fomentar el trabajo en grupo: En este sentido, se busca romper el círculo de confort del alumnado mezclando estudiantes de grados distintos sin experiencia previa en trabajos en común. Esto implica, por un lado, una importante labor de control por parte del profesorado

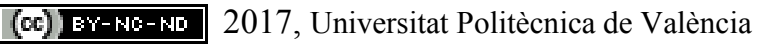


y una disponibilidad adicional para resolver las dudas logísticas y de contenido que puedan ir surgiendo, así como para organizar los talleres a los que acudirán ambos grupos de manera conjunta. Pero por otro, genera grandes beneficios, ya que fomenta el intercambio de ideas entre alumnos con intereses e inquietudes distintos, pero también coincidentes en algunos casos. Al mismo tiempo, esta mezcla consigue que los estudiantes se acerquen a ámbitos del mundo de las letras que no les son del todo conocidos, entrando en contacto directo con las ideas de un escritor en el caso de los traductores, o acercándose al mundo de la traducción y sus complejidades en el de los autores.

\section{Desarrollo de la innovación}

\subsection{Requisitos previos y necesidades}

Antes de comenzar con el grueso del proyecto, fue necesario dar varios pasos. En primer lugar, establecer los recursos humanos y materiales a utilizar en el desarrollo de esta actividad. Entre los primeros, basta contar con los cuatro docentes implicados y un número de alumnos mínimo que haga viable el proyecto. Entre los segundos, las necesidades son escasas: las copias necesarias de poemas y traducciones, un aula con ordenador donde celebrar los encuentros entre escritores y traductores y alguna herramienta telemática que facilite la comunicación entre los participantes. En este caso, se emplea la plataforma virtual Blackboard de la que dispone la Universidad de Alcalá.

Este espacio virtual optimiza el trabajo en varios sentidos. En primer lugar, permite subir materiales como los poemas originales, las distintas versiones de las traducciones o los materiales empleados en los talleres para que los alumnos los consulten en cualquier momento. Asimismo, facilita la comunicación telemática entre las partes, ya que permite asegurar que las convocatorias para los talleres y otras actividades relacionadas son recibidas por el total de los participantes. Asimismo, crea una red cerrada de mensajes en la que cualquier miembro del proyecto puede contactar con otro vía correo electrónico cuando lo desee.

Sin embargo, dentro del amplio abanico de recursos que ofrece Blackboard, la herramienta cuya utilización conviene destacar especialmente es Blackboard Collaborate, un entorno virtual que permite dividir a los participantes en distintos grupos y en el que los alumnos pueden trabajar de forma telemática en tiempo real. Con este recurso, poetas y traductores pueden intercambiar archivos a la vez que interactúan con sus compañeros, bien a través de video conferencia, bien de forma escrita en el chat habilitado a tal efecto. Por otro lado, Blackboard Collaborate permite a los profesores monitorizar el trabajo de los estudiantes cuando este se está llevando a cabo, así como realizar presentaciones de contenido en tiempo real que luego pueden guardarse para el acceso a posteriori de los participantes no conectados en ese momento. En definitiva, este recurso simplifica significativamente las labores de coordinación de alumnos y profesorado, ya que los estudiantes siempre disponen de un entorno propio al que conectarse sin necesidad de reunirse físicamente, algo que resulta especialmente importante cuando el grupo lo componen alumnos procedentes de distintas partes de la Comunidad de Madrid. Y lo hace 
además a través de una interfaz sencilla e intuitiva que los alumnos de ambos grados rápidamente comprendieron tras unas breves explicaciones en el segundo taller presencial.

Otro aspecto a preparar de antemano fue el reparto de tareas entre los docentes participantes. En este sentido, se determinó que el profesor Bruno Echauri asumiese las labores de organización y coordinación del proyecto, la profesora Maya García la de proporcionar las explicaciones teóricas necesarias sobre el proceso de traducción llevado a cabo por los alumnos y el comentario que debían desarrollar al respecto, y la profesora Silvia García la de preparar y analizar los cuestionarios finales completados por los alumnos. Estos tres profesores se encargaron también de seleccionar los poemas y conducir los distintos talleres. Por su parte, el profesor Jonathan Sell asumió las labores de revisión y edición de los poemas dado el valor añadido y la perspectiva que aporta al proyecto que un profesor nativo se encargue de esta fase del trabajo. De este modo, se consigue una mayor naturalidad en el texto meta y se pueden identificar e incluir u omitir matices que a profesores de nacionalidad española se les escapen.

En cuanto a los plazos de trabajo y las fechas límite, ambos vinieron condicionados fundamentalmente por el calendario académico. Puesto que la asignatura de Traducción Literaria, Edición y Corrección de Textos se imparte a lo largo del primer cuatrimestre, este periodo generaba automáticamente la ventana de tiempo en la que trabajar con los alumnos. Por ello, el proyecto se puso en marcha al poco de comenzar el curso, con sesiones iniciales para determinar el número de participantes o dar las primeras instrucciones, entre otros. A continuación, se planificaron tres talleres presenciales comunes para los alumnos de EEHH como de LLMMyT a completar poco después de las vacaciones navideñas puesto que se necesitaba tiempo para evaluar los resultados finales e incluirlos en la calificación de los alumnos de traducción. Terminado el cuatrimestre, comenzó un proceso final de revisión y edición por parte del profesor Jonathan Sell para preparar los poemas para su publicación. Todos y cada uno de estos pasos se detallan en el siguiente apartado.

\subsection{Fases}

El primer encuentro con los alumnos afectó únicamente al grupo de EEHH. En este taller, se aportaron más detalles sobre los poemas: se fijó una fecha de presentación y se acordó que cada autor debía hacer entrega 3 o 4 poemas de estilo libre centrados en el siguiente tema: (in)experiencias. Este tópico da gran libertad al alumno y le invita transmitir sus vivencias y lo que le queda por vivir, abriéndose al lector e interiorizando que este es en gran medida su trabajo, por y para ellos.

Recopilados los poemas, se seleccionaron los que formarían parte de la antología, para lo cual se tuvieron en cuenta distintos criterios. Por un lado, la calidad literaria. Por otro, la dificultad de los textos, sobre todo en base a las capacidades del grupo de traductores. En este sentido, hemos tenido la fortuna de contar con un grupo de LLMMyT que posee un alto nivel de capacidades y conocimientos, por lo que el margen en lo que a complejidad de los textos se refiere fue bastante amplio. Otras variables a tener en cuenta

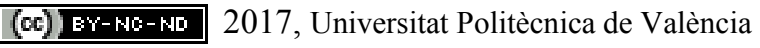


fueron la cantidad y longitud de los poemas. Ambas tienen como misión cumplir dos objetivos. Por un lado, no sobrecargar a los alumnos con una carga de trabajo excesiva. Por otro, reunir una cantidad de poemas suficiente para crear una antología bilingüe adecuada para una publicación. En base a todos estos criterios, los poemas escogidos finalmente fueron 23 sobre un total de 34 .

Hecho esto, se establecieron los emparejamientos, para lo cual se recuperan tres de los parámetros anteriores: capacidad del traductor, longitud y cantidad de poemas. Siguiendo estos criterios se buscaba encontrar un equilibrio entre dificultad y volumen de los textos a traducir. Finalmente, surgieron 8 grupos: 4 parejas, dos tríos de dos traductores y un escritor, otro trío a la inversa y un cuarteto formado por dos escritores y dos traductores. A continuación, tuvo lugar el primer taller conjunto.

En este encuentro, autores y traductores se conocieron por primera vez personalmente. Posteriormente, se organizaron los grupos de trabajo y se entregaron los poemas correspondientes. Durante este taller, se dio tiempo a los traductores para realizar una primera lectura y plantear sus dudas, tanto a "sus" autores en particular como al resto de compañeros. A esta ronda de preguntas siguió un debate en el que se intentaron aportar distintas soluciones (véase apartado 4). Como trabajo para la siguiente sesión, se pidió que preparasen un primer borrador y corrigiesen los posibles errores del original.

Finalmente, se presentaron las utilidades de la plataforma virtual y de la herramienta Blackboard Collaborate. A pesar de que muchos alumnos la desconocían, el manejo fácil e intuitivo de esta aplicación hace que sea relativamente sencillo familiarizarse con ella. Como se mencionaba anteriormente, Blackboard Collaborate permite dividir a los alumnos en distintos grupos para que puedan trabajar de manera independiente (con o sin la supervisión del profesor). Asimismo, ofrece la posibilidad de mantener videoconferencias, chatear o intercambiar archivos y mantener tutorías en línea en cualquier momento. Herramientas como esta facilitan sobremanera la consecución de proyectos de este tipo en el que se involucran personas de distintos grados, con distintos horarios y responsabilidades que resulta complicado conjugar. Dado que Blackboard también ofrece la posibilidad de colgar archivos para su posterior descarga, el profesorado sube a esta plataforma todos los poemas originales al concluir el taller; de este modo, todos los estudiantes del grupo pueden, en cualquier momento, leerlos si así lo desean.

El tercer encuentro con los alumnos se centró en las principales dificultades encontradas al elaborar el primer borrador, dudas a las que sus compañeros, y el profesorado en última instancia, sugirieron posibles soluciones. También se procedió a la lectura en voz alta de algunos de los poemas, un paso que en ocasiones puede pasarse por alto pero que permite medir la idoneidad del texto meta en distintos aspectos: ritmo, efectos sonoros, puntuación, etcétera. Tener al lado al escritor original supone una enorme ventaja, ya que se trata de la persona que más interiorizada tiene la sonoridad del poema y, por consiguiente, es la más indicada para orientar al traductor en la difícil tarea de encontrar una armonía sonora relativamente similar. 
Posteriormente, se mostró al grupo un esquema con los puntos a tratar en el comentario sobre la traducción que están realizando: estilo del autor, elementos de variación y registro, prosodia, rima u ortotipografía con función dramática, entre otros. Esta parte se orienta fundamentalmente a los traductores, aunque los escritores deben también realizar una labor de apoyo a los primeros en caso de que necesiten resolver alguna duda. Este esquema cumple además otra función en el taller: mostrar a los alumnos puntos de la traducción en los que podían no haber reparado hasta entonces y, de este modo, 1) abrir un debate en torno a ellos y 2) repasar y perfeccionar las traducciones teniendo estos puntos en cuenta. A destacar, elementos como la ortotipografía o el tono según la intención dramática, factores que en ocasiones son relegados a un segundo plano pero cuya adecuada traducción no se puede obviar si queremos lograr un texto meta de alto valor literario. Finalmente, se anunció que para el siguiente encuentro, los alumnos deberán haber concluido la traducción de los poemas y el comentario.

El último taller comenzó con una nueva lectura en voz alta de textos originales y meta. Posteriormente, con la perspectiva que otorga haber terminado ya el trabajo, se comentaron las principales dificultades encontradas a la hora de realizar las traducciones y elaborar el comentario al respecto.

También se buscó conocer de viva voz la experiencia por parte de los alumnos a lo largo del proyecto, a fin de identificar los puntos fuertes y débiles del proyecto y preparar posibles mejoras para darle continuidad de distintas formas en el futuro. Asimismo, se busca su parecer con respecto a la posibilidad de realizar esta actividad en cursos venideros, incidiendo en temas como la utilidad de la tarea, el grado de aprendizaje alcanzado con ella o el interés que les ha despertado, todos ellos con respuestas muy positivas. A la conclusión del taller, se entrega a todos los participantes un cuestionario a este respecto que deberán rellenar por escrito.

Finalizado el trabajo con los alumnos, comienza el proceso de edición por parte del profesorado, a cargo del profesor Jonathan Sell. En este sentido, se buscó la colaboración de un profesor nativo para dotar al texto meta de la mayor naturalidad posible y descubrir, si los hubiera, errores que a los que tienen el inglés como segunda lengua puede costar más identificar. Conviene recalcar a este respecto que no hubo cambios significativos en ningún poema, lo que también prueba la calidad de las traducciones realizadas. Revisados y editados, los poemas se subieron a la plataforma para que los alumnos los consultasen y se abrió un periodo de resolución de dudas en el que pudieron contactar con el profesor Jonathan Sell para preguntar cualquier cosa relacionada con este último proceso de revisión y edición.

Al margen de las fases anteriores, también resulta fundamental el trabajo fuera del aula, tanto por parte del profesorado como, lógicamente, por parte de los alumnos. Entre los cometidos de los primeros se cuentan labores de coordinación, supervisión y orientación como la preparación de todo el material necesario para los talleres, monitorización del aula virtual y del trabajo en línea de los alumnos, asistencia a desdobles cuando alguno de los grupos de trabajo no puede asistir a alguno de los talleres o tutorías para resolver las dudas de los alumnos. Cabe destacar que pasadas las primeras semanas, en las que los alumnos de

(cc)) EY-NC-ND 2017, Universitat Politècnica de València 
EEHH plantearon alguna duda sobre la elaboración de los poemas, este recurso fue aprovechado principalmente por los alumnos de LLMMyT con el objetivo de pulir sus traducciones o responder a ciertas preguntas relacionadas con la elaboración del comentario que debían preparar al final.

Los alumnos, por su parte, deben completar varias horas de trabajo autónomo en las que no participa el profesorado. Para ello, cada grupo ha utilizado los recursos a su disposición en la medida en que ha creído más oportuno. Al margen de las herramientas propuestas por la universidad, los alumnos emplearon también otros mecanismos para comunicarse entre ellos: grupos de Whatsapp, herramientas como Google Drive, videoconferencias o, por supuesto, el correo electrónico. El contacto directo varió en función de las posibilidades de cada grupo, pero conviene subrayar el consenso en torno a su utilidad y valor. La posibilidad de este contacto directo entre autores y traductores es una de las grandes ventajas de este proyecto y un motivo más de satisfacción para todos los participantes.

\section{Resultados}

Para evaluar los resultados obtenidos, remitimos al lector al apartado 2, puesto que intentaremos medir el éxito del proyecto en base a la consecución de los objetivos establecidos anteriormente. En primer lugar, el resultado más tangible de todo el proyecto ha sido un poemario bilingüe pendiente de publicación del que se adjuntan dos ejemplos:

ARDIENDO.

Tu sonrisa egocéntrica se ha tornado de colores ceniza.

Has quemado todos los botones de tu camisa $\mathrm{y}$ ahora me pides que te arrope con mi cuerpo.

Las sílabas se han calcinado en tu lengua, pero tus te quiero aún arden debajo del paladar.

Tu pecho sube y baja al compás de las llamaradas.

Arden tus ojos.

Los mismos ojos que un día fueron agua.
BURNING.

\section{Your egocentric smile}

is now coloured ash.

you have burnt all the buttons of your shirt and now you are asking me to wrap you up with my body.

The syllables have charred in your tongue but your I love you's are still burning under the palate.

Your chest goes up and down to the beat of the flashes.

Your eyes are burning.

The same eyes that once were water. 
Tú también eres humano

No mires a tus infiernos,

has salido de ellos

dejando la puerta abierta,

pero eres afortunado en la huida

el fuego ha dejado huella en tu gesto,

derretido la plata para sueños,

evaporado las ansias del amor.

Has sufrido la pérdida,

descubierto la soledad,

saboreado la impotencia.

Hoy Orfeo estás de enhorabuena;

eres humano y perderás la cabeza por ello.
You're human too

Don't look at your circles of hell, you made it out alive leaving an open gate,

But you are fortunate in your escape: hellfire left a mark on your face, melt the daydreams' dough, and burnt off the thirst for love. You suffered from loss, discovered solitude and savoured helplessness.

Orpheus, you're in luck today; you're human and it will cost your head.

Para medir aspectos como la motivación, el desarrollo del trabajo en grupo, el manejo de la segunda lengua y otros factores incluidos en el proyecto, los alumnos participantes completaron un cuestionario en función del grado al que pertenecían, con los siguientes resultados:

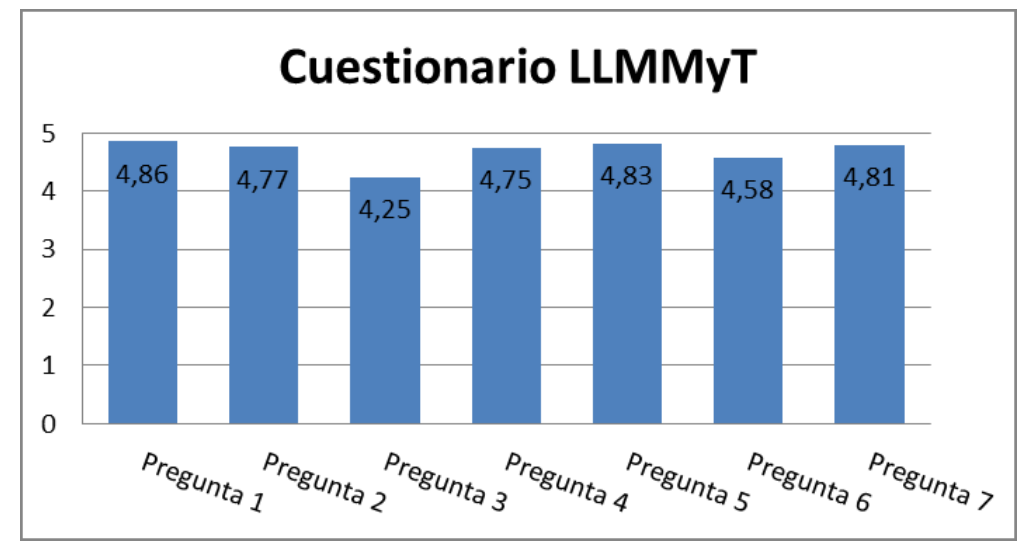

Pregunta 1: Fomento del interés por la traducción literaria y motivación por seguir en contacto con esta disciplina.

Pregunta 2: Utilidad de la iniciativa para identificar y resolver problemas relacionados con la traducción poética

Pregunta 3: Manejo y utilidad de herramientas informáticas Aula Virtual y Blackboard Collaborate

Pregunta 4: Grado de satisfacción con el trabajo con personas de una disciplina distinta a la tuya

Pregunta 5: Grado de ayuda al trabajar con el autor del poema que has traducido

Pregunta 6: Grado de satisfacción con los talleres presenciales

Pregunta 7: Utilidad de la iniciativa para la motivación y la mejora de la visión de la experiencia universitaria 


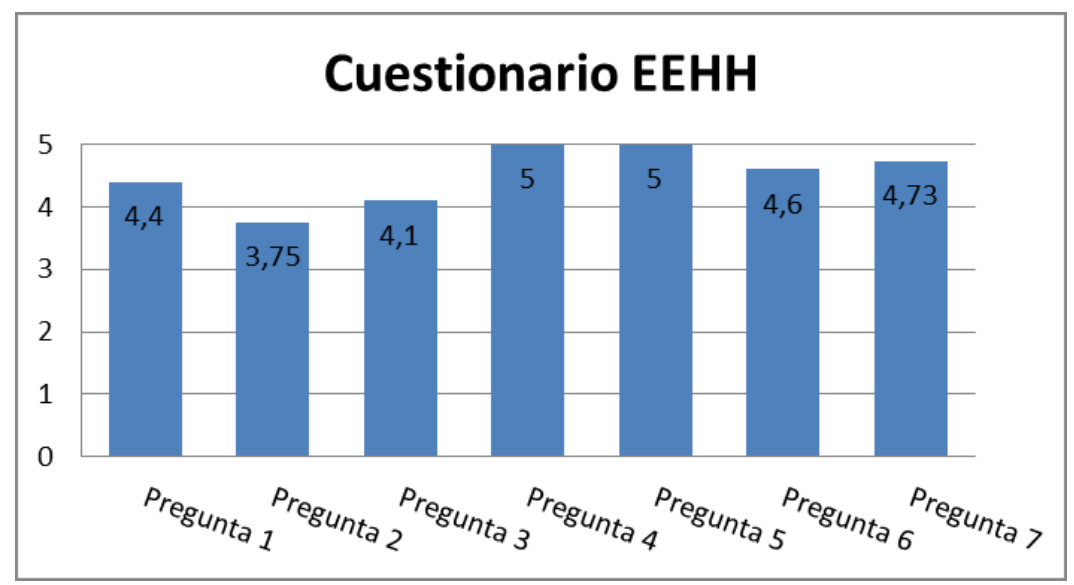

Pregunta 1: Fomento del interés por la el inglés como L2 y motivación por seguir en contacto con esta lengua. Pregunta 2: Utilidad de la iniciativa como un estímulo a tu creatividad.

Pregunta 3: Manejo y utilidad de herramientas de herramientas informáticas Aula Virtual y Blackboard Collaborate Pregunta 4: Grado de satisfacción con el trabajo con personas de una disciplina distinta a la tuya

Pregunta 5: Grado de satisfacción al trabajar con el traductor de tu obra.

Pregunta 6: Grado de satisfacción con los talleres presenciales.

Pregunta 7: Utilidad de la iniciativa para la motivación y la mejora de la visión de la experiencia universitaria

El análisis de los resultados cualitativos de los comentarios de los alumnos de LLMMyT mostró una satisfacción mayoritaria con la experiencia y coinciden en que ha fomentado su interés por la traducción literaria, su motivación para seguir en contacto con esta disciplina y su capacidad para resolver problemas relacionados con la traducción poética.

El trabajo transversal con alumnos de otro grado les ha resultado muy positivo y enriquecedor. A este respecto, muchos destacan la posibilidad de colaborar con el autor original y cómo esto les ha permitido resolver dudas, trasladar connotaciones adecuadamente y ganar confianza a la hora de traducir sus poemas. También resaltan la buena relación con los alumnos de EEHH y las nuevas ideas y perspectivas que estos les han aportado. Aunque en menor medida, también valoran positivamente su experiencia con la herramienta Blackboard Collaborate, destacando las posibilidades que les ha ofrecido la hora de compartir material y poder comunicarse en tiempo real de manera virtual. Sin embargo, también señalan fallos puntuales en el sistema que han obstaculizado su trabajo en contadas ocasiones.

Por último, en lo que respecta a su nivel de satisfacción con este tipo de iniciativas, existe unanimidad en las respuestas de todos los estudiantes, que han valorado la pregunta 
con la máxima puntuación. Todos los traductores consideran que su experiencia en la participación a lo largo de este proyecto ha sido muy motivadora y que ha mejorado su visión de la experiencia universitaria. De la misma manera, comentan que este proyecto ha sido una práctica muy positiva para su formación como futuros traductores e incluso algunos han manifestado su interés en seguir formándose en el campo de la traducción literaria. Por último, en sus comentarios agradecen haber tenido la oportunidad de participar en una actividad que fomenta la creatividad de los alumnos.

Los resultados de los cuestionarios de EEHH muestran que la participación de estos alumnos en el proyecto ha aumentado su interés por la lengua inglesa. Asimismo, muchos consideran que su motivación por seguir en contacto con el inglés se ha incrementado, y apuntan también que actividades de este tipo suponen un estímulo para su creatividad y les animan a seguir trabajando es sus creaciones propias.

El grado de satisfacción referente al trabajo con compañeros de otro grado es también muy elevado. Los escritores definen la experiencia, al igual que sus compañeros de LLMMyT, como muy enriquecedora y subrayan que este proyecto les ha permitido acercarse a sus poemas desde una perspectiva distinta: la de los traductores. Asimismo, coinciden en destacar este tipo de actividades como un modo de mejorar la experiencia universitaria, puesto que se trata de una forma diferente y emocionante de acercarse a la enseñanza en la que sus propias creaciones y opiniones juegan un papel fundamental.

Por último, en lo referente al manejo de herramientas informáticas como Blackboard y sus distintas utilidades, los alumnos de EEHH coinciden con los traductores en que, a pesar de problemas esporádicos, su capacidad para utilizar estos recursos ha mejorado.

En cuanto a las pautas orientadas a mejorar la enseñanza de la traducción poética, se ha trabajado con los comentarios de los alumnos de LLMMyT así como con las preguntas que han ido surgiendo en los distintos encuentros. A continuación, se presentan algunos de los puntos conflictivos a los que se dio solución ${ }^{2}$ y que serán tenidos en cuenta a la hora de mejorar la docencia en próximos cursos:

I) Priorizar forma o contenido, respetar la métrica, pérdida de significado: Estas tres son dudas recurrentes de los alumnos participantes en el proyecto. La respuesta puede basarse en contrastar dos posibilidades. La primera sería seguir un enfoque tradicional, basado en una reproducción fiel del poema original en la que se sigue un modelo traductológico palabra por palabra que elimina la creatividad del traductor del proceso. Esta línea es fácilmente descartable si se muestran traducciones literales de varios poemas que se hayan trasladado a la lengua española en comparación con otras alternativas.

Por otro lado, existe otra aproximación a este tipo de traducción, basada en la ya clásica premisa de Benjamin (1994) de mantener el sentido a través de la libertad en la traducción. A este punto de partida creemos conveniente añadir las palabras de Eco (2004),

\footnotetext{
${ }^{2}$ Los puntos conflictivos y pautas para abordarlos son más, pero por motivos de espacio se han incluido únicamente algunos ejemplos representativos.
}

(c)) BY-NC-ND 2017, Universitat Politècnica de València 
quien defiende alejarse del sentido literal para trascender el texto de partida y tratar de recrearlo en otra lengua a través de un equilibrio entre fidelidad al poema y reinvención del mismo, lo cual requiere un rol más activo por parte del traductor.

Como describe el párrafo anterior, esta última corriente no debe interpretarse como el contrario a la primera. En nuestra opinión, el objetivo de la traducción poética debe ser tratar de crear un efecto similar al del original, lo que no implica un nivel de fidelidad preestablecido, sino una búsqueda de la similitud a través de la reproducción y no del calco. Para conseguirlo, debe enseñarse al traductor que fidelidad y creatividad no son excluyentes en una misma tarea, sino que puede moverse entre ambos polos. En este sentido, es importante sistematizar el proceso. Grosso modo, lo que deberá hacer en primer lugar será identificar la acústica, la métrica, la rima, el empleo de lenguaje y la retórica que contiene el original. Una vez identificadas y analizadas y a fin de alcanzar ese efecto cercano al del escritor, el traductor deberá escoger qué aspectos mimetizar, cuáles pasar a segundo plano y cuáles modificar. Para esto, el traductor no puede verse encorsetado por normas rígidas, sino que es necesaria cierta dosis de creatividad y un margen de maniobra que los enfoques en los que equivalencia significa máxima fidelidad al original no permiten.

II) Traducción de expresiones idiomáticas y registros coloquiales: Estas dudas pueden ligarse con otros aspectos léxicos y semánticos relevantes a la hora de afrontar la traducción de un poema: neologismos, falsos amigos o dialectos, entre otros. En este sentido, una buena práctica a enfatizar para guiarnos en la traducción es la de estudiar la obra del autor para descubrir el uso que este suele hacer del lenguaje, tratar de comprender lo que se esconde detrás y, eventualmente, trazar paralelismos entre otros poemas del mismo escritor y aquel a cuya traducción nos enfrentamos (Gómez-Pato, 2012). En este caso, la presencia del autor original evita esta tarea de investigación, pero conviene subrayar esta directriz como algo necesario en la mayor parte de los casos.

En lo que atañe a expresiones idiomáticas, refranes y casos similares conviene señalar al traductor novel que lo habitual será tratar de encontrar equivalentes en la lengua meta al tiempo que se comprueba si el autor ha respetado o alterado estas estructuras; a continuación, el traductor deberá imitar esta práctica con su equivalente en español para conservar el efecto del original (Gómez-Pato, 2012). Una vez más, conocer las tendencias del autor en este sentido y si hace un uso convencional o rupturista del lenguaje puede resultar extremadamente útil.

\section{Conclusiones}

A través de este proyecto de innovación docente se pretendía encontrar una manera innovadora de hacer partícipes a los alumnos de su propio proceso de aprendizaje, en el que pudieran ser protagonistas y no meros receptores de información, a través de una metodología novedosa y colaborativa.

Como se deriva de los resultados del proyecto y del grado de satisfacción de los alumnos (y profesores) participantes, los objetivos planteados en un principio se han cumplido satisfactoriamente. Con el desarrollo del proyecto, hemos conseguido estimular 
la creatividad de los alumnos, tanto de los creadores de los poemas originales como de los traductores como re-creadores de los poemas en lengua inglesa. Asimismo, como los propios alumnos han indicado, la motivación para continuar en contacto con la lengua inglesa en el caso de los alumnos de EEHH y de la traducción literaria en el caso de los de LLMMyT ha mejorado, así como su visión general de la experiencia universitaria.

Sin embargo, no sólo se ha conseguido motivar y promover la creatividad de los alumnos, sino que también, a través de este proyecto diferente y novedoso, se ha fomentado el trabajo en grupo entre alumnos de grados distintos con excelentes resultados, introduciendo las nuevas herramientas tecnológicas como Blackboard Collaborate para facilitar el contacto entre ellos y el intercambio de ideas y documentos, dándoles la posibilidad de trabajar en cualquier momento y lugar desde cualquier dispositivo conectado a internet.

Por último, conviene recalcar el alto grado de transferibilidad de este proyecto, si no en su totalidad, al menos parcialmente. Los pocos recursos materiales necesarios, la flexibilidad de grados con los que se puede trabajar (al menos en el lado de los escritores) y el balance coste-beneficios que generan, hacen que experiencias como la descrita en las páginas anteriores supongan una mejora en el proceso de enseñanza y de aprendizaje para todas las partes implicadas.

\section{Referencias}

- BENJAMIN, W. (1994). La tarea del traductor. En M.A. Vega, Textos clásicos de teoría de la traducción, 285-296. Madrid: Cátedra.

- CHAÍN-NAVARRO, C., MARTÍNEZ-SOLÍS, L. y SÁNCHEZ-BAENA, J.J. (2008). "Motivar desde la innovación en la enseñanza universitaria: El blog Qalidad". RED: Revista de Educación a Distancia, 21.

- DE LA TORRE, S. (2009). "La universidad que queremos: estrategias creativas en el aula universitaria". Revista Digital Universitaria, 10(12). Disponible en http://www.revista.unam.mx/vol.10/num12/art89/art89.pdf

- ECO, U. (2004). Experiences in translation. Toronto: University of Toronto Press.

- GERVILla CASTILlO, A. (2003). Creatividad aplicada. Una apuesta de futuro. Madrid: Dykinson.

- GÓMEZ-PATO, R.M. (2012). Apuntes para una didáctica de la traducción de poesía / notes on poetry translation and teaching. Teoría De La Educación: Educación y Cultura En La Sociedad De La Información, 13(1), 236-265.

- POSEY, M. (2009). The advantages of L2 translation in The Cat in the Hat: A closer look at translation directionality. Letras, 46, 87-100.

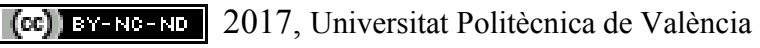

